\title{
Broadband over Power Line used in Intelligent Residential District
}

\author{
Y.T. Zhou, X.M. Wang, W. Deng, S. Chen, M. Wang \\ State grid Chongqing electric power companyNanan power branch \\ Chongqing, China
}

\begin{abstract}
Intelligent Residential District has been common life, as the digital life develops. Broadband over Power Line plays an important role in intelligent residential district, for its communication reliability and easy-constructed. The paper analyses the traits of Broadband over Power Line, introduces the technology how to be used in intelligent residential district, and values the effects.
\end{abstract}

Keywords-intelligent residential district; broadband over power line; communication

\section{INTRODUCTION}

\section{A. Smart Grid and Intelligent Power Consumption}

Smart grid research originated in the United States, then some other countries endorsed the view, its goal is to realize the smart grid in 2020 or the more distant future.

State Grid Corporation dedicated to provide clean energy, promote economic development, to serve society as the basic mission. Carefully analyzed the new trend of world power grid development and China's national condition, combining the new situation of China's energy supply and the new demand of electricity service, proposed based on independent innovation, according to the principle of unified planning, unified standards, unified construction, constructed the UHV power grid as the backbone, coordinated all levels of power grid, with information technology, automation, interactive features of the smart grid development goals.

Intelligent power is one of the important pillars of construction of strong smart grid, the basis and physical carrier for achieving all kinds of strong smart grid functions as well as the standpoint of strong smart grid construction.

\section{B. Intelligent Residential District}

The construction of intelligent residential district is a measure of intelligent power consumption to further deepen and implement, as well as the most powerful foothold for intelligent power consumption. To carry out pilot work construction of intelligent residential district is to study how smart grid advanced technology apply in residential areas, explore the intelligent residential district construction mode, show the smart grid results of state grid corporation. Then to disseminate strong smart grid advanced concepts, improve the level of intelligent power grid, as well as improve service ability, the pilot of the new business model, promote the development of intelligent residential district in order.

Intelligent residential district will centrally embody the characteristics of intelligent power consumption includes advanced technology, efficient economy, service diversity, flexible interaction, friendly open. Adopting advanced communication technology such as optical fiber composite cable and power line carrier, constructing communication network coverage the district. Building a two-way communication channel between Power Grid Corp and consumer in intelligent residential district, to solve the electricity information acquisition, community intelligent power distribution automation, 95598 community service interactive platform, distributed energy access control, electric vehicle charging and orderly management of energy efficiency, smart home and other issues, as well as provide users with two-way interactive service and triple play service, establish intelligent power saving mode, realize low carbon environmental protection, safe and comfortable, convenient and fast power supply service, actively explore area of low voltage power distribution management and new operation mode of power supply services.

\section{The Intelligent Residential District Construction in Chongqing}

According to the urban construction guidelines, unified planning, rational layout, comprehensive development and construction matching, and relevant requirements of intelligent residential construction, Chongqing electric power company selected Fubaoquan Community and Jiaxinqinyuan Community in northern New District of Chongqing for the pilot area through detailed investigation and careful consultation with other departments.

The construction area of Fubaoquan Community is 123400 square meters, the pilot involving 806 households, using PLC technology to collect customer electricity information. While the Jiaxinqinyuan Community 99100 square meters involving 528 households, it is a new district, using PFTTH and EPON technology to collect customer electricity information. Both communities are high-rise residential with a large population, populous, electricity situation is complex, and it can represent the characteristics of residents district in Chongqing. At the same time, the two communities are more high-grade modern buildings, facilities, suitable for intelligent electricity pilot project, and have good demonstration and leading effect.

\section{BROADBAND OVER POWER LINE [1-3]}

Power Line Communication (PLC) refers to directly use the power line as a communication medium, coupling carrier signal in power line and modulating to realize data communication. At present, the highest communication rate of 
Broadband over Power Line (BPL) can reach 200 Mbps. BPL uses advanced OFDM modulation mode that has strong channel adaptive ability and anti-interference ability. BPL is generally used in indoor in the United States and Japan, while it can be used in outdoor and indoor in Europe. At present, BPL has been used in electric information acquisition system pilot in state grid corporation.

The network structure of 200M power line communication system is a master-slave network, there is a master node in the network, and the other PLC terminal as slave nodes, all slave nodes can't directly communicate with each other, the communication among them through the master node to complete.

\section{A. Function Characteristics}

Orthogonal frequency division multiplexing modulation technology has high transmission efficiency and good antijamming performance [4].

Master-slave communication mode improve the utilization rate of bandwidth and easy to management.

DES/3 DES mixed encryption to fully guarantee the safety of the data.

Support the simple network management protocol and remote software update.

Support 802.1Q VLAN and OVLAN network control protocol.

Provide QoS (quality of service) level control [5-6].

\section{B. Key Technology}

(1) Coding and modulation method

- 1536 ways sub carrier of OFDM modulation

- m-QAM sub carrier modulation

- Adaptive sub carrier modulation technique

- $\quad$ R-S forward error correction coding

- Interleaved code

- 4D grid code

\section{(2) Adaptive OFDM channel}

In order to guarantee a certain bit error rate, SNR threshold of carrier for different modulation rate is different. The lower modulation rate is the lower SNR threshold.

(3) Media access control (MAC) with QoS

200 Mbps PLC can be allocatedtothe corresponding channel resource according to the different service needs of users.

(4) Programmable power spectral density and spectral notching

For some reason (such as EMC, and other equipment of deposit), there needs to be a spectrum of carrier power spectrum density control within a certain range, may even need to shut it down.
TABLE I. TECHNICAL INDICATORS.

\begin{tabular}{|l|l|}
\hline Maximum physical layer rate & $200 \mathrm{Mbps}$ \\
\hline Frequency range & $1-34 \mathrm{MHz}$ \\
\hline Carrier channels & 1536 \\
\hline Maximum modulation ratio & $9 \mathrm{bit} / \mathrm{sec} / \mathrm{Hz}$ \\
\hline Signal dynamic range & $90 \mathrm{~dB}$ \\
\hline Minimum received power & $-70 \mathrm{~dB}$ \\
\hline Output power step value & $1 \mathrm{~dB}$ \\
\hline Power spectral density & $-50 \mathrm{dBm} / \mathrm{Hz}$ \\
\hline Typical time delay & $2 \mathrm{~ms}$ \\
\hline Typical error rate & $10^{-9}$ \\
\hline
\end{tabular}

\section{APPLICATION OF BROADBAND OVER POWER LINE IN THE INTELLIGENT COMMUNITY}

In the construction of intelligent community, the Fubaoquan Community use BPL technique, it is mainly embodied in the electric information acquisition, as shown in Fig. 1.

The data acquisition system still adopt three layers structure of the general set copy system, including the main station layer, data acquisition terminal layer, meter layer, wherein the remote channel using optical fiber communication technology, the local channel using concentrator and broadband carrier collector and 485 electric energy meter to collecting meter information.

Installing broadband concentrator at residential distribution transformer, directly collecting distribution transformer inspection table via the 485 interface while collecting lowvoltage residential single/three-phase electric energy meter and common commercial industrial customers electric energy meter through the collector.Residential customers every three layers shareone collector, contiguous general industry and commerce customer share a collector.



FIGURE I. APPLICATION OF BPLC IN THE INTELLIGENT COMMUNITY.

The collector using broadband carrier to upload data to the district distribution indoor concentrator which will send the district electricity information to main station, thus realize the residential electricity information acquisition.

\section{BROADBAND OVER POWER LIN TECHNOLOGY APPLICATION EFFECT EVALUATION}

Broadband over Power Line, as a contemporary the most advanced power line communication mode, has the advantages of low cost, stable signal and fast transmission speed, so it can well meet the needs of the remote set copy system on lower level communication.

According to the characteristics of Broadband over Power 
Line, the 806 households of Fubaoquan Community successfully realized their electricity information acquisition and the success rate can reach $100 \%$.

In the construction the system, Broadband over Power Line technology embodies the following characteristics:

(1) Make full use of the existing low voltage power line resources, saving the cost of construction, accelerate the construction progress

(2) Power line communication using orthogonal frequency division multiplexing (OFDM) modulation technology, improves the anti-interference ability of data transmission and the frequency utilization ratio

(3) From the collector to the background station full use the TCP/IP protocol, the metering accuracy is high, the transmission speed is fast.

(4) System uses all kinds of equipment, which are suitable for Chongqing with high temperature and high humidity environment.

(5) It has advantage of transmission bandwidth, besides can meet the remote collecting can also be used for Internet access, VOIP, and provide high-speed data transmission platform for power load monitoring, demand side management, intelligent household and digital communities.

Low Voltage Broadband over Power Line technology with low cost, high transmission speed, high stability, powerful management and other advantages, overcomes the disadvantages of domestic meter reading field and stands out from the numerous meter reading systems. It has become one of the most cost-effective solutions for electric power construction enterprises, and has great popularization value and application prospect.

\section{REFERENCE}

[1] Liu W, Sigle M, Dostert K. Channel characterization and system verification for narrowbandpower line communication in smart grid applications[J]. Communications Magazine, IEEE,2011, 49(12): 28-35.

[2] Kim I H, Varadarajan B, Dabak A. Performance analysis and enhancements of narrowband OFDM powerline communication systems[C]//Smart Grid Communications (SmartGridCom), 2010 First IEEE International Conference on. IEEE, 2010: 362-367.

[3] Umari M H, Razazian K, Petrovska O. A novel preamble for OFDM symbol synchronizationthat can outperform PN-based preambles in narrowband channels[C]//Circuits and Systems(MWSCAS), 2010 53rd IEEE International Midwest Symposium on. IEEE, 2010: 777-780.

[4] Rieken D W. Periodic noise in very low frequency power-line communications[C]//Power LineCommunications and Its Applications (ISPLC), 2011 IEEE International Symposium on. IEEE,2011: 295-300.

[5] Katayama M, Yamazato T, Okada H. A mathematical model of noise in narrowband power linecommunication systems[J]. Selected Areas in Communications, IEEE Journal on, 2006, 24(7):1267-1276.

[6] Tao Z, Xiaoxian Y, Baohui Z, et al. Research of noise characteristics for 10-kVmedium-voltage power lines[J]. Power Delivery, IEEE Transactions on, 2007, 22(1): 142-150. 